\title{
Performance comparison of aspirin assay between Anysis and VerifyNow: Assessment of therapeutic platelet inhibition in patients with cardiac diseases
}

\author{
Jinxiang Piao ${ }^{\mathrm{a}}$, Chaeyoung Yoo $^{\mathrm{a}}$, SeonYoung Kim ${ }^{\mathrm{b}}$, Youn-Wha Whang ${ }^{\mathrm{c}}$, \\ Cheol Ung Choi ${ }^{\mathrm{c}, *}$ and Sehyun Shin ${ }^{\mathrm{a}, \mathrm{d}, *}$ \\ ${ }^{a}$ Engineering Research Center for Biofluid Biopsy, Korea University, Seoul, Korea \\ ${ }^{\mathrm{b}} R \& D$ Center, Rheomeditech. Inc., Seoul, Korea \\ ${ }^{\mathrm{c}}$ Division of Cardiology, Department of Internal Medicine, College of Medicine, Korea University, \\ Seoul, Korea \\ ${ }^{\mathrm{d}}$ School of Mechanical Engineering, Korea University, Seoul, Korea
}

\begin{abstract}
.
BACKGROUND: Assessment of platelet inhibition for aspirin therapy is important to manage patients who are at potential risk of developing thrombotic and hemorrhagic complications.

OBJECTIVE: This study aimed to evaluate a new platelet assay (Anysis-aspirin), compare it with VerifyNow-aspirin in patients with cardiac diseases, and analyze the aspirin resistance rates between the two devices.

METHODS: Citrated blood samples were collected from patients with cardiac diseases referred for the aspirin response test. In the Anysis assay, a test result was provided with a blood flow migration distance (MD) until blood flow stoppage, which was comparable to aspirin reaction units (ARUs) obtained using VerifyNow. The measurements were simultaneously conducted using the two devices and compared.

RESULTS: The MD without and with aspirin use was $160 \pm 33$ and $254 \pm 23 \mathrm{~mm}$, respectively $(p<0.0001)$. Compared with VerifyNow (reference), the sensitivity and specificity of Anysis-200 were 96.3 and $90.3 \%$, respectively (area under the curve, 0.968). Furthermore, the aspirin resistance rate in aspirin-administered patients was $20.9 \%$ using VerifyNow and $16.5 \%$ for Anysis-200. The Cohen's kappa coefficient between the two devices was 0.81 , indicating an almost perfect agreement between the two devices.

CONCLUSIONS: Anysis-aspirin, a novel aspirin assay for assessing platelet inhibition, showed excellent agreement with VerifyNow-aspirin with high accuracy and precision. The Anysis-aspirin assay would be used as a point-of-care test to assess aspirin non-responsiveness and abnormal platelet reactivity.
\end{abstract}

Keywords: Platelet function, aspirin, Anysis-200 analyzer, VerifyNow

\footnotetext{
*Corresponding authors: Cheol Ung Choi, MD, Ph.D., Division of Cardiology, Department of Internal Medicine, Guro Hospital, Korea University College of Medicine, Seoul 06273, South Korea. E-mail: wmagpie@korea.ac.kr; Sehyun Shin, Ph.D., School of Mechanical Engineering, Korea University, Seoul 02841, South Korea. Tel.: +82 232903377 ; Fax: +82 2 928 5825; E-mail: lexerdshin@korea.ac.kr.
} 


\section{Introduction}

Platelets are involved in many pathological processes, such as vascular tone regulation, inflammation, host defense, and tumor biology. Among the multiple functions of platelets, the major concern is the development of thrombosis, which is the main cause of mortality. Recent studies have reported that platelets are also intricately involved in immune functions during viral infection, including severe acute respiratory syndrome coronavirus 2 (SARS-CoV-2). Activated platelets corresponding to viral infection result in platelet aggregation, microthrombosis, and reduced platelet count [1-3]. Antiplatelet therapy is recommended for improving the ventilation/perfusion ratio in patients with severe respiratory failure from COVID-19 [4]. A clinical study on the effect of platelet reactivity on mortality in patients with cardiovascular diseases receiving antiplatelet agents reported that the hyperreactive platelet response results in higher CVD events rather than a low-reactive platelet response [5]. Also, hyperreactive platelet aggregation has been an important factor on the restenosis rate after stenting [6]. Due to the increase in the incidence of cardiovascular diseases associated with platelet reactivity, antiplatelet drugs, such as aspirin and clopidogrel, are widely used as treatment and preventive agents [7,8]. Therefore, there is a general need to test the degree of platelet inhibition when taking antiplatelet drugs.

In response to these demands, several platelet assays are now available for examining antiplatelet therapeutic responses, such as the Platelet Function Analyzer-200 (Siemens, Mississauga, Ontario, Canada), Multiplate Analyzer (Roche Diagnostics, Rotkreuz, Switzerland), and VerifyNow (Accumetrics, San Diego, CA, USA). Each of them has adopted its unique technique to assay platelet aggregation levels. For instance, VerifyNow measures the transmitted light intensity as a measure of inhibitory effect on platelet aggregation of drug (aspirin and P2Y12 inhibitor) with respect to time. These devices have been validated with a reference method reporting excellent agreement and proven useful in identifying patients with abnormal responses to antiplatelet therapy [9, 10]. However, surprisingly, a study on the performance comparison between these three assays yielded poor agreement results [11]. More seriously, the clinical outcomes did not agree with the measured results [12].

The Anysis-200 analyzer (Rheo Meditech Inc., Seoul, Korea) has been introduced as a point-of-care (POC) device for platelet function testing and assessment of antiplatelet therapeutic response [13-16]. Anysis-200 has been demonstrated with its usefulness for screening patients with platelet dysfunction, showing substantial agreement with VerifyNow [16-19]. Anysis-200 is an automated microfluidic assaying system that mimics an in vivo thrombosis-inducing environment. Anysis-200 provides the migration distance (MD) of blood through a microchannel as a measure of adhesion and aggregation of platelets, which is comparable to the aspirin resistance units (ARUs) of VerifyNow. The objective of the present study is to evaluate the performance of the Anysis-200 system with VerifyNow in screening patients with aspirin-resistance.

\section{Materials and methods}

\subsection{Patient samples}

A total of 142 whole blood samples were collected at the Korea University Guro Hospital between April 2020 and December 2020. The present study adopted following exclusion criteria: hematocrit $<35 \%$ and $>60 \%$, platelet count $<100 \times 10^{9} / \mathrm{L}$, abnormal value of either prothrombin time or activated partial thromboplastin time within the previous month, pregnancy, and use of anticoagulation agents. The samples from patients who were not taking aspirin were defined as negative controls, whereas those from patients who took aspirin were defined as positive controls. The study protocol was approved by the Institutional Review Board of Korea University Guro Hospital (IRB No. 2019GR0390), and written consent was obtained from all patients prior to enrollment in the study. 
Blood samples were collected in 3.2\% sodium citrate tubes in both the Anysis-200 and VerifyNow assays. After blood collection, the tubes were gently inverted (5-6 times). Special care was taken to avoid agitation-induced platelet activation during transportation. Then, the blood samples were left still at room temperature $\left(\sim 23^{\circ} \mathrm{C}\right)$ for $30 \mathrm{~min}$ prior to testing. All tests were completed within $120 \mathrm{~min}$ after blood collection to minimize the risk of time-dependent platelet malfunction [20].

The operating principles of Anysis-200 and VerifyNow were described in our previous study [16]. In brief, Anysis-200 measures platelet aggregation in a stenosis-mimetic microtube. Upstream-activated platelets flow through a tube and adhere to the fibrinogen-coated microbeads, recruit aggregate with each other, and eventually block the flow passages in the microtube [13-15]. When the blood flow is completely blocked, Anysis-200 provides the migration distance (MD) of the blood as a measure of platelet function. If platelets are inhibited by aspirin, the MD will be increased. MD values > $204 \mathrm{~mm}$ were considered abnormal or indicative of aspirin-inhibited platelet function. Meanwhile, VerifyNow has been widely used for anti-platelet assessment. In a test chamber, activated platelets tend to form clusters with fibrinogen-coated microbeads and transmitted light intensity variations with time reports in ARUs. Abnormal ARUs were defined as $<550$, according to the manufacturer's instructions. Any values $<550$ were considered abnormal or indicative of aspirin-inhibited platelet function [18].

\subsection{Statistical analysis}

Normally distributed data are expressed as the mean \pm standard deviation (SD). The sensitivity and specificity were analyzed considering the results from VerifyNow as true positive and negative values. The true negative controls were the samples derived from patients who did not take aspirin and an ARU value of 550 or higher $(n=62)$, whereas the true positives were the samples from patients who took aspirin and an ARU value of less than $550(n=80)$. The agreement between the two assays was examined using Cohen's kappa coefficient [21]. Statistical significance was set at $p<0.05$. In the present statistical analyses, MedCalc version 12.1.4 software (MedCalc Software, Mariakerke, Belgium) was used.

\section{Results}

\subsection{Descriptive characteristics and hematologic parameters}

Among the total of 142, 96 male and 46 female patients, aged $65.6 \pm 9.2$ years (mean $\pm 1 S D$ ), were included in the study (Table 1). Among them, 51, 54, and 78 patients were diagnosed with hypertension, diabetes mellitus, and dyslipidemia, respectively. 38 (26.8\%) patients took aspirin alone, and 42 patients took both aspirin and P2Y12 inhibitor. Among the total aspirin taking samples, 19 of them showed aspirin-resistance with VerifyNow and thus were excluded for sensitivity analysis. The clinical characteristics of the patients are summarized in Table 1.

\subsection{Comparative measurements between the two platelet function assays}

In the present analysis, normal (or negative) control was defined as patients who did not take aspirin and yielded $A R U \geq 550$, whereas positive group was defined as the patients who took aspirin and yielded ARU $<550$ (Table 2). The mean value in the negative control group $(n=62)$ was $160.5 \pm 33.4 \mathrm{~mm}$, whereas that in patients using aspirin (true positives, $n=80$ ) was $254.5 \pm 23.3 \mathrm{~mm}$ $(p<0.0001)$. Similarly, VerifyNow-aspirin showed ARU values of $618.2 \pm 31.9$ for true negative controls and $450.5 \pm 51.6$ for the true positive group $(p<0.0001)$. 
Table 1

Baseline patient characteristics

\begin{tabular}{|c|c|}
\hline Variable & Overall $(\mathrm{N}=142)$ \\
\hline Age (years), mean \pm SD & $65.6 \pm 9.20$ \\
\hline Male sex, n (\%) & $96(67.6 \%)$ \\
\hline \multicolumn{2}{|l|}{ Risk factors } \\
\hline Hypertension, n (\%) & $51(35.6 \%)$ \\
\hline Diabetic mellitus, n (\%) & $54(38.0 \%)$ \\
\hline Hyperlipidemia, n (\%) & $78(54.9 \%)$ \\
\hline \multicolumn{2}{|l|}{ Medicines } \\
\hline Aspirin, n (\%) & $38(26.8 \%)$ \\
\hline P2Y12 inhibitors, $\mathrm{n}(\%)$ & $29(20.4 \%)$ \\
\hline DAPT, n (\%) & $42(29.6 \%)$ \\
\hline \multicolumn{2}{|l|}{ Laboratory findings } \\
\hline $\mathrm{RBCs}\left(\times 100^{3} / \mu \mathrm{L}\right)$ & $4.4 \pm 0.4$ \\
\hline WBCs $\left(\times 100^{3} / \mu \mathrm{L}\right)$ & $6.8 \pm 2.3$ \\
\hline Platelets $\left(\times 100^{3} / \mu \mathrm{L}\right)$ & $213.9 \pm 50.1$ \\
\hline Hemoglobin (g/dL) & $13.4 \pm 1.3$ \\
\hline Hematocrit (\%) & $41.5 \pm 3.8$ \\
\hline PT (s) & $12.9 \pm 0.6$ \\
\hline aPTT (s) & $33.8 \pm 4.6$ \\
\hline Glucose (mg/dL) & $105.1 \pm 18.5$ \\
\hline
\end{tabular}

Continuous data are shown as mean $\pm 1 \mathrm{SD}$.

Table 2

Comparison of migration distances (a) between negative controls and patients using aspirin and (b) between before and after aspirin use

\begin{tabular}{llll}
\hline & Groups & Mean \pm SD & $p$-value \\
\hline (a) Anysis & Negative controls $(n=62)$ & $160.5 \pm 33.4$ & $<0.0001$ \\
& Patients using aspirin $(n=80)$ & $254.5 \pm 23.3$ & \\
(b) VerifyNow & Negative controls $(n=62)$ & $618.2 \pm 31.9$ & $<0.0001$ \\
& Patients using aspirin $(n=80)$ & $450.5 \pm 51.6$ & \\
\hline
\end{tabular}

Through the present clinical comparison test, Anysis-200 showed 96.3\% (95\% CI, 89.4-99.2\%) of the sensitivity and $90.3 \%$ (95\% CI, 80.1-96.4\%) of specificity, respectively (Fig. 1). The present study further examined the receiver operating characteristic (ROC) curve for comparison of Anysis200 with VerifyNow (Fig. 2). The area under the curve (AUC) for Anysis-aspirin was 0.968 with a cutoff value of $205 \mathrm{~mm}(p<0.001)$. Additionally, among the patients who took aspirin $(n=91), 19$ $(20.9 \%)$ and $15(16.5 \%)$ patients showed ARUs $\geq 550$ using VerifyNow and MD $\leq 204$ using Anysis200, respectively (Table 3). The correlation between VerifyNow and Anysis-200 was significantly high (Pearson's correlation coefficient, $r=-0.73, p<0.0001$ ). According to the Cohen's kappa coefficient $(\kappa)$, the agreement rate between Anysis-200 and VerifyNow was 0.81 (which was interpreted as excellent agreement between two devices. 


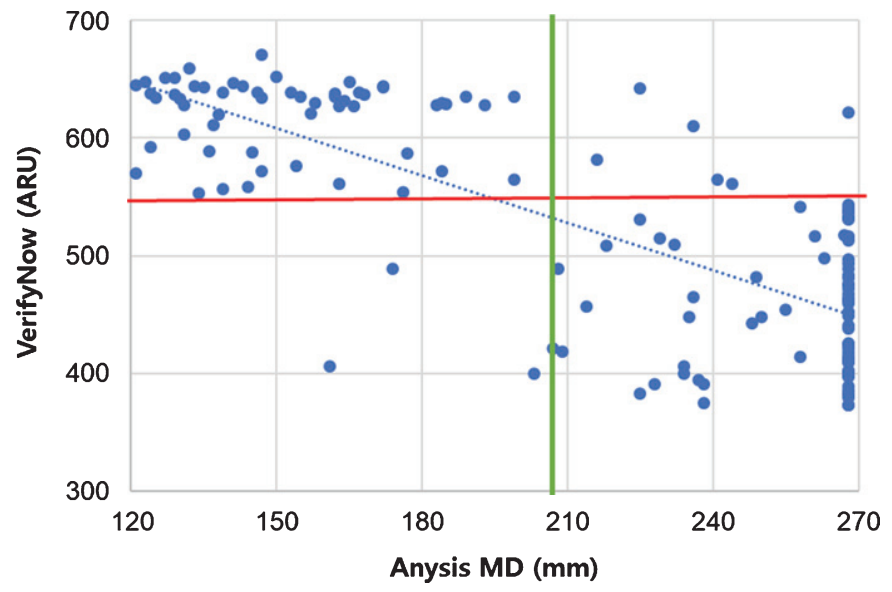

Fig. 1. Scatter plot comparing Anysis-200 and VerifyNow.

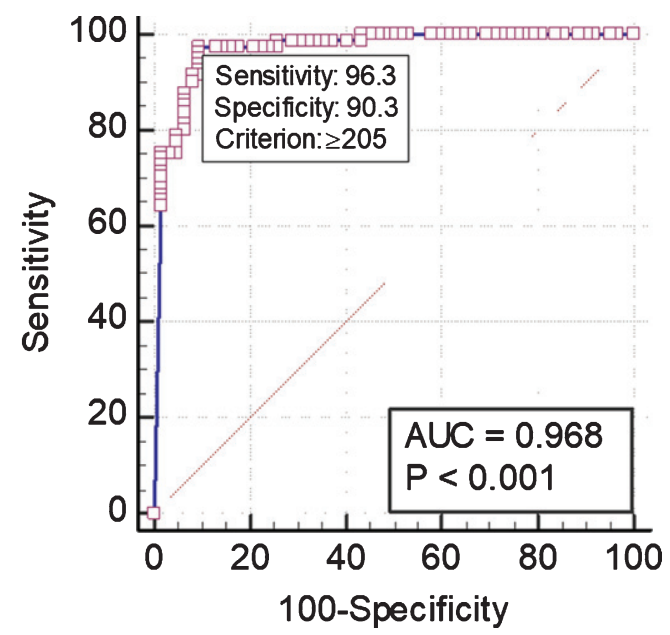

Fig. 2. Receiver operating characteristic curve comparing Anysis-200 and VerifyNow.

Table 3

Comparison of aspirin non-responsiveness between (a) VerifyNow and (b) Anysis

\begin{tabular}{lccc}
\hline & Cut-off & $\begin{array}{c}\text { Rates of aspirin- } \\
\text { resistance }\end{array}$ & $\begin{array}{c}\text { Agonist } \\
\text { concentration }\end{array}$ \\
\hline (a) VerifyNow & $\mathrm{ARU} \geq 550$ & $20.9 \%(19 / 91)$ & $1 \mathrm{mM}$ \\
(b) Anysis-200 & $\mathrm{MD}<205 \mathrm{~mm}$ & $16.5 \%(15 / 91)$ & $0.144 \mathrm{mM}$ \\
\hline
\end{tabular}

\section{Discussion}

In our previous study, the Anysis-200 system demonstrated the same performance as VerifyNow [18] with a yielding sensitivity of $91.5 \%$ and specificity of $75.5 \%$. In the present study, the specificity of Anysis-200 was further increased to $90.3 \%$ by adopting the strict definition of negative controls. Owing to the increase in specificity, the Cohen's kappa coefficient $(\kappa)$ was also significantly increased to 0.81 , which indicates almost perfect agreement with the reference device. 
Table 4

Various indexes to representing diagnostic performance

\begin{tabular}{|c|c|c|c|}
\hline & Anysis-aspirin & & Definition \& classification \\
\hline Sensitivity (\%) & 96.3 & & True positives/(true positive+false negatives) \\
\hline Specificity $(\%)$ & 90.3 & & True negatives/(true negative+false positives) \\
\hline Sen + Spe $(\%)$ & 186.6 & OK & Sensitivity + specificity $\geq 1.5(150 \%)$ \\
\hline \multirow[t]{3}{*}{ AUC } & 0.968 & $\sqrt{ }$ & AUC > 0.9: high accuracy \\
\hline & & & $0.7 \sim 0.9:$ moderate accuracy \\
\hline & & & $0.5 \sim 0.7:$ low accuracy \\
\hline \multirow[t]{3}{*}{ LR+ } & 9.93 & & Definition: sensitivity/(1 - specificity) \\
\hline & & & $>10$ : having potential to alter clinical decisions \\
\hline & & $\sqrt{ }$ & $\begin{array}{l}5 \sim 10 \text { : providing useful additional information } \\
<5 \text { : rarely to alter clinical decisions }\end{array}$ \\
\hline \multirow[t]{4}{*}{ LR- } & 0.10 & & Definition: (1 - sensitivity)/specificity] \\
\hline & & $\sqrt{ }$ & $\leq 0.1$ : having potential to alter clinical decisions \\
\hline & & & $0.11 \sim 0.20$ : providing useful additional information \\
\hline & & & $0.21 \sim 3$ : rarely to alter clinical decisions \\
\hline \multirow[t]{2}{*}{ Diagnostic odd ratio } & 99.3 & OK & Definition: $(\mathrm{LR}+/ \mathrm{LR}-)$ \\
\hline & & & $\mathrm{DOR}>20$ \\
\hline
\end{tabular}

The VerifyNow system has been widely applied to monitor the effectiveness of antiplatelet agents, such as aspirin and P2Y12 inhibitor, in various clinical environments [9, 10]. However, VerifyNow has major drawbacks, including the high cost per test and hematocrit dependence. The most serious problem is the disagreement between test results and clinical outcomes, including aspirin resistance. As listed in Table 3, aspirin resistance (AR) was found to be $20.9 \%$ in VerifyNow, and similar results were also reported in previous studies [18]. However, the AR of Anysis was 16.5\%, which was slightly lower than that of VerifyNow. However, somewhat different results were reported for the P2Y12 assay in which low response rates of P2Y12 inhibition therapy were 36.5\% in VerifyNow and 5.9\% in Anysis. [19].

Even though there is no agreed universal definition of aspirin resistance, it can be interpreted as either "clinical aspirin resistance" or "in vitro aspirin resistance" [22, 23]. The former describes patients who experience vascular complications despite aspirin therapy [24, 25], whereas the latter is associated with method-dependent AR associated with agonist concentrations and aspirin dose. The problem is that these two ARs cannot be differentiated until clinical outcomes occur. First, it would be worthwhile to examine the effect of agonist concentrations on the low response rate to antiplatelet therapy. The concentrations of arachidonic acid (AA) were $1 \mathrm{mM}$ in VerifyNow and $0.144 \mathrm{mM}$ in Anysis. Since the concentration of AA in VerifyNow is 7-fold higher than that in the Anysis, a normal dose (100 mg) of aspirin may not sufficiently block the receptor from external AA-induced activation. Thus, the LRR of aspirin obtained using the VerifyNow system might be relatively high compared to that obtained using the Anysis assay. Second, it is worth noting that the initial low responder rate $(15 \%)$ was significantly reduced to 5\% when the aspirin dose was doubled [26]. Considering these results, antiplatelet assays should minimize the concentration of agonists to avoid false-positive results of aspirin resistance.

In Table 4, we examined the diagnostic performance of the present Anysis-aspirin with various indexes. First, the sums of sensitivity and specificity are $186.6 \%$, which is much higher than the threshold value of $150 \%$. Second, the value of AUC is 0.968 , which is ranked in the highest regions of accuracy. Third, LR + is 9.93, which showed second highest rank with providing useful additional information and LR- is 0.10 , which is ranked in the highest rank having potential to alter clinical 
decisions. The diagnostic odds ratio for Anysis-aspirin is 99.3, which is much higher than the 20. All these indexes revealed that the Anysis-aspirin assay has the equivalent level of diagnostic performance of VerifyNow-aspirin assay.

Conclusively, the present study confirmed that the performance of the newly developed platelet function assay, Anysis-aspirin, was equivalent to that of VerifyNow-aspirin in terms of sensitivity and specificity. Owing to the adoption of innovative technologies, Anysis-aspirin is one of the most user-friendly antiplatelet assays that can provide rapid and precise results in any clinical environment. Furthermore, the proposed antiplatelet assay can significantly improve diagnosis by identifying patients who do not respond adequately to platelets inhibiting drugs to prevent thrombosis and replace drugs with other effective treatments.

\section{Conflicts of interest}

The authors declare no conflicts of interest.

\section{Acknowledgments}

This research was supported by the Seoul R\&D Project (no. HI14C0670).

\section{Ethical approval}

This study was approved by the Institutional Review Board, Korea University Guro Hospital (approval no. 2019GR0390) and was performed in accordance with the Declaration of Helsinki. Informed consent was obtained from all participants in the study.

\section{References}

[1] Wool GD, Miller JL. The Impact of COVID-19 Disease on Platelets and Coagulation. Pathobiology. 2021;88(1):15-27. doi: 10.1159/000512007

[2] Estevez B, Du X. New Concepts and Mechanisms of Platelet Activation Signaling. Physiology (Bethesda). 2017;32(2):162-77.

[3] Braune S, Küpper JH, Jung F. Effect of Prostanoids on Human Platelet Function: An Overview. Int J Mol Sci. 2020;21(23):9020. doi: 10.3390/ijms21239020.

[4] Viecca M, Radovanovic D, Forleo GB, Santus P. Enhanced platelet inhibition treatment improves hypoxemia in patients with severe Covid-19 and hypercoagulability. A case control, proof of concept study. Pharmacol Res. 2020;158:104950

[5] Parodi G, Marcucci R, Valenti R, Gori AM, Migliorini A, Giusti B, et al. High Residual Platelet Reactivity After Clopidogrel Loading and Long-term Cardiovascular Events Among Patients With Acute Coronary Syndromes Undergoing PCI. JAMA. 2011;306(11):1215-23.

[6] Bach R, Jung F, Kohsiek I, Ozbek C, Spitzer S, Scheller B, Dyckmans J, Schieffer H. Factors affecting the restenosis rate after percutaneous transluminal coronary angioplasty. Thromb Res. 1994;74(Suppl 1):S55-67.

[7] Reinthaler M, Braune S, Lendlein A, Landmesser U, Jung F. Platelets and coronary artery disease: Interactions with the blood vessel wall and cardiovascular devices. Biointerphases. 2016;11(2):029702.

[8] Ullrich H, Gori T. The pleiotropic effects of antiplatelet therapies. Clin Hemorheol Microcirc. 2019;73(1):29-34.

[9] Welsh KJ, Dasgupta A, Nguyen AN, Wahed A. Utility of VerifyNow for Point-of-Care Identification of an Aspirin Effect Prior to Emergency Cardiac Surgery. Ann Clin Lab Sci. 2015;45(4):377-81.

[10] Chatterton S, Dignan R, Luu Q, Aty W, Chandrasiri S, French JK. Platelet Activity Measured by VerifyNow® Aspirin Sensitivity Test Identifies Coronary Artery Bypass Surgery Patients at Increased Risk for Postoperative Bleeding and Transfusion. Heart Lung Circ. 2020;29(3):460-8. 
[11] Kim J, Cho CH, Jung BK, Nam J, Seo HS, Shin S, et al. Comparative evaluation of Plateletworks, Multiplate analyzer and Platelet function analyzer-200 in cardiology patients. Clin Hemorheol Microcirc. 2018;70(3):257-65.

[12] Van Werkum JW, Harmsze AM, Elsenberg EHAM, Bouman HJ, Ten Berg JM, Hackeng CM. The use of the VerifyNow system to monitor antiplatelet therapy: a review of the current evidence. Platelets. 2008;19(7):479-88.

[13] Song SH, Lim CS, Shin S. Migration distance-based platelet function analysis in a microfluidic system. Biomicrofluidics. 2013;7(6):64101.

[14] Song SH, Lim CS, Shin S. Scalable evaluation of platelet aggregation by the degree of blood migration. Appl Phys Lett. 2013;103:243702.

[15] Lee H, Kim G, Lim C, Lee B, Shin S. A simple method for activating the platelets used in microfluidic platelet aggregation tests: stirring-induced platelet activation. Biomicrofluidics. 2016;10(6):064118. doi: 10.1063/1.4972077.

[16] S Xu, J Piao, BK Lee, CS Lim, S Shin, Platelet thrombus formation by upstream activation and downstream adhesion of platelets in a microfluidic system. Biosens. Bioelectron 2020;165:112395.

[17] Park SJ, Yoon J, Seo HS, Lim CS. Performance evaluation of the Anysis-200 platelet function analyzer in cardiac patients. Clin Hemorheol Microcirc. 2020. doi: 10.3233/CH-190801.

[18] YH Chung, KA Lee, M Cho, S Shin, BK Lee, Performance comparison of platelet function analyzers in cardiology patients: VerifyNow and Anysis-200 aspirin assays. Clin Hemorheol Microcirc. 2020;76(1):33-42.

[19] Jinxiang Piao, Seon Young Kim, Youn-Wha Whang, Cheol Ung Choi, Sehyun Shin, Assessment of therapeutic platelet inhibition in cardiac patients: Comparative study between VerifyNow-P2Y12 and Anysis-P2Y12 assay. Clin Hemorheol Microcirc. doi: 10.3233/CH-211104.

[20] Braune S, Walter M, Schulze F, Lendlein A, Jung F. Changes in platelet morphology and function during 24 hours of storage. Clin Hemorheol Microcirc. 2014;58(1):159-70.

[21] McHugh ML. Interrater reliability: the kappa statistic. Biochem Med (Zagreb). 2012;22(3):276-82.

[22] Muir AR, McMullin MF, Patterson C, McKeown PP. Assessment of aspirin resistance varies on a temporal basis in patients with ischaemic heart disease, Heart. 2008;95(15):1225-9.

[23] van Oosterom N, Barras M, Bird R, Nusem I, Cottrell N. Drugs. A Narrative Review of Aspirin Resistance in VTE Prophylaxis for Orthopaedic Surgery. 2020;80(18):1889-99.

[24] Hankey GJ, Eikelboom JW. Aspirin resistance. BMJ. 2004;328(7438):477-9.

[25] Patrono C. Aspirin resistance: definition, mechanisms and clinical read-outs. J Thromb Haemost. 2003;1(8):1710-3.

[26] Sibbing D, Braun S, Morath T, Mehilli J, Vogt W, Schömig A, et al. Platelet reactivity after clopidogrel treatment assessed with point-of-care analysis and early drug-eluting stent thrombosis. J Am Coll Cardiol. 2009;53(10):849-56. 